Proc. Budapest'02 Workshop on

Quark $\&$ Hadron Dynamics (2002) 000-000

\begin{tabular}{l}
\hline \hline Budapest'02 Workshop on \\
Quark \& Hadron Dynamics \\
Budapest, Hungary \\
March 3-7, 2002 \\
\hline \hline
\end{tabular}

\title{
Partonic Effects in Heavy Ion Collisions at RHIC
}

\author{
C. M. Ko, Zi-wei Lin, and Subrata Pal \\ Cyclotron Institute and Physics Department, Texas A\&M University, \\ College Station, Texas 77843-3366
}

\begin{abstract}
Effects of partonic interactions in heavy ion collisions at RHIC are studied in a multiphase transport model (AMPT) that includes both initial partonic and final hadronic interactions. It is found that a large parton scattering cross section is needed to understand the measured elliptic flow of pions and two-pion correlation function.
\end{abstract}

Keywords: transport model, parton scattering, elliptic flow, pion interferometry PACS: 25.75.-q,25.75.Dw,25.75.Gz,25.75.Ld

\section{Introduction}

In heavy ion collisions at RHIC, a quark-gluon plasma is expected to be created in the initial stage. Many observables have been suggested as possible signals for the quark-gluon plasma. These include enhanced production of dileptons [ 1 ] and strange hadrons [ 2], suppression of $J / \psi$ production [ 3], Hanbury-Brown-Twiss interferometry between pions [ [4], elliptic flow of various particles [5], quench of jet production [ [6], and fluctuations in the net charge [ 7], net baryon [ []] as well as total baryon numbers [ 9]. However, to establish these observables as unambiguous signals for the quark-gluon plasma requires that they are not affected appreciably by the final hadronic dynamics in the collisions. To study quantitatively these signals, we have developed a multiphase transport model [10] that includes both the dynamics of initial partonic and final hadronic matters. With this model, we have studied not only the rapidity and transverse momentum distributions of various charged particles [ 11] but also their elliptic flows [ 12]. Furthermore, we have investigated multistrange baryon production [ 13], $J / \psi$ suppression [ 14] and pion interferometry [15] in these collisions. We find that the model describes very well the experimental data on the charged particle rapidity distributions and transverse momentum spectra. However, these results are more sensitive to the hadronic interactions than the partonic interactions. On the other hand, both the charged particle elliptic flow and the two-pion correlation function are affected by partonic interactions, and to explain the observed large elliptic flow and the measured pion 
correlation function in the experiments requires a large parton-parton scattering cross section in the initial partonic matter.

\section{Multiphase Transport Model}

In the AMPT model, the initial conditions are obtained from the HIJING model [ 16] by using a Woods-Saxon radial shape for the colliding nuclei and including the nuclear shadowing effect on parton production via the gluon recombination mechanism of Mueller-Qiu [17. After the colliding nuclei pass through each other, the Gyulassy-Wang model [18] is then used to generate the initial space-time information of partons. In the default HIJING model, these minijet partons are allowed to lose energy via the gluon splitting mechanism and transfer their energies to the nearby strings associated with initial soft interactions. Such a jet quenching is replaced in the AMPT model by explicitly taking into account parton-parton collisions via Zhang's Parton Cascade (ZPC) [ 19]. At present, only gluon elastic scatterings with a default cross section of $3 \mathrm{mb}$ are included, so the partons do not suffer any inelastic energy loss as they traverse the dense matter. After partons stop interacting, they combine with their parent strings and, after an average proper formation time of $0.7 \mathrm{fm} / \mathrm{c}$, are converted to hadrons using the Lund string fragmentation model [20]. Dynamics of the resulting hadronic matter is then described by a relativistic transport model (ART) [ 21], which has been improved to include baryon-antibaryon production from meson-meson interactions and their annihilation [22].

The parameters in the AMPT model are determined by fitting the experimental data from $\mathrm{Pb}+\mathrm{Pb}$ collisions at center-of-mass energy $\sqrt{s}=17 \mathrm{~A} \mathrm{GeV}$ at SPS [ 23]. Specifically, to describe the measured net baryon rapidity distribution, we have included in the Lund string fragmentation model the popcorn mechanism for baryon-antibaryon production with equal probabilities for baryon-meson-antibaryon and baryon-antibaryon configurations. Also, to account for both the pion yield and the enhanced kaon yield in this reaction, we have adjusted the two parameters in the momentum splitting function used in the Lund string fragmentation model, following the expectation that the string tension is modified in the dense matter formed in the initial stage of heavy ion collisions.

Since the probability for minijet production is very small in heavy ion collisions at SPS energies, the partonic stage does not play any role in these collisions. We find that final-state hadronic scatterings reduce the proton and antiproton yields, but increase kaon and antikaon production by about $20 \%$. In contrast, the kaon yield in the default HIJING model is smaller than our final result by about $40 \%$.

\section{Rapidity and Transverse Momentum Distributions}

Results on the charged particle rapidity distributions in central $\mathrm{Au}+\mathrm{Au}$ collisions at RHIC are shown in the left four panels of Fig. 1 for center-of-mass energies of 
$56 A \mathrm{GeV}$ (dashed curves) and $130 \mathrm{~A} \mathrm{GeV}$ (solid curves) together with data from the PHOBOS collaboration [24]. The measured total charged particle multiplicities at mid-pseudorapidity at both energies are well reproduced by our model. Also, the $\bar{p} / p$ ratio predicted by the AMPT model is consistent with that measured in experiments [25]. The transverse momentum spectra of pions, kaons, and protons in $\mathrm{Au}+\mathrm{Au}$ collisions at $\sqrt{s}=130 \mathrm{~A} \mathrm{GeV}$ are shown in the right panel of Fig. 1 . The results from the the AMPT model are seen to also agree with the experimental data from the PHENIX collaboration [26].
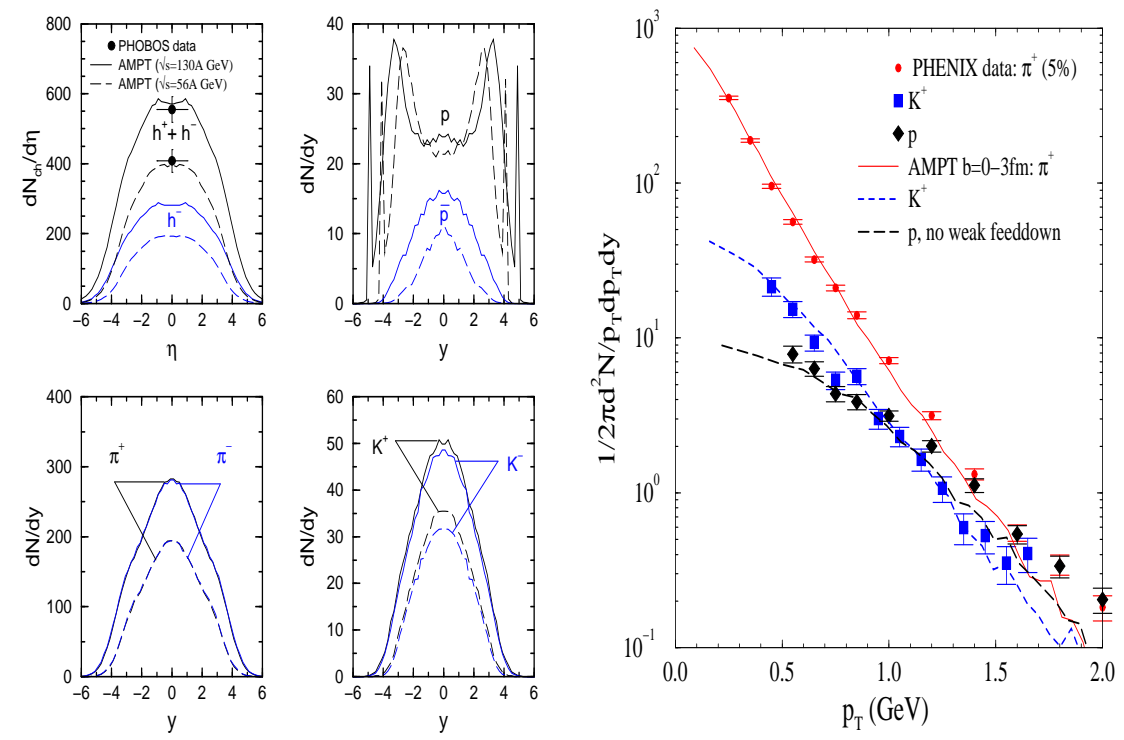

Fig. 1. Left four panels: Rapidity distributions of total and negatively charged particles, protons and antiprotons, charged pions, and charged kaons in $\mathrm{Au}+\mathrm{Au}$ collisions at $\sqrt{s}=56 \mathrm{~A}$ and $130 \mathrm{~A} \mathrm{GeV}$. Right panel: Transverse momentum spectra of pions, kaons, and protons in $\mathrm{Au}+\mathrm{Au}$ collisions at $\sqrt{s}=130 \mathrm{~A} \mathrm{GeV}$. The experimental data are for $5 \%$ most central collisions from the PHOBOS collaboration [24] for rapidity distributions and from the PHENIX collaboration [26] for transverse momentum spectra. Results from the AMPT model shown by curves are for impact parameters of $b \leq 3 \mathrm{fm}$.

Furthermore, the AMPT model describes very well the centrality dependence of the charged particle rapidity distribution measured by the BRAHMS collaboration [27] as shown in Fig. 2 by dashed curves. Compared to the default HIJING model given by solid curves, hadronic scatterings are responsible for the broader rapidity distributions seen in the experiments.

Without hadronic interactions, there is a significant increase in the numbers of total charged particles, pions, protons, and antiprotons at midrapidity. The 

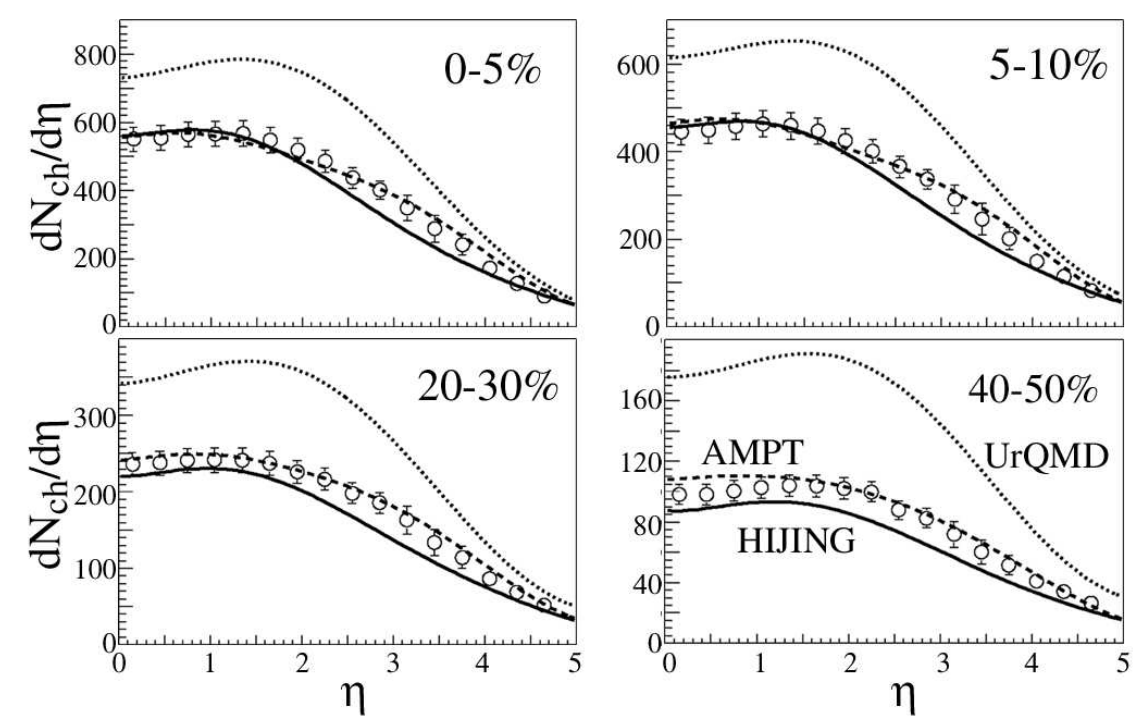

Fig. 2. Charged particle rapidity distributions for centralities of 0-5\%, 5-10\%, 20$30 \%$, and $40-50 \%$ in $\mathrm{Au}+\mathrm{Au}$ collisions at $\sqrt{s}=130 \mathrm{~A} \mathrm{GeV}$. The experimental data are from the BRAHMS collaboration [27]. Theoretical results from the AMTP model are shown by dashed curves while those from the default HIJING model are given by solid curves. The figure is taken from Ref.[27].

kaon number is, on the other hand, reduced slightly. As a result, the ratios of $\bar{p} / p$ and $K^{+} / \pi^{+}$in the absence of final-state hadronic interactions are 0.80 and 0.13 , respectively, instead of 0.66 and 0.18 from the default AMPT model, which are more consistent with the data. Although the default HIJING [ 28] gives a total charged particle multiplicity at midrapidity that is consistent with the PHOBOS data, including hadronic scatterings would reduce its prediction appreciably.

Effects of partonic dynamics on final hadronic observables can be studied in the AMPT model by turning off the partonic cascade. We find that this leads to less than $\sim 5 \%$ change in the final charged particle yields in $\mathrm{Au}+\mathrm{Au}$ collisions at $\sqrt{s}=130 \mathrm{~A} \mathrm{GeV}$. The multiplicity distribution of hadrons is thus not very sensitive to parton elastic scatterings.

\section{Elliptic Flow}

Elliptic flow in heavy ion collisions measures the anisotropy of particle momentum distributions in the plane perpendicular to the beam direction. It results from the initial spatial anisotropy in non-central collisions and is thus sensitive to the properties of the hot dense matter formed during the initial stage of heavy ion 
collisions [ 5, 29]. In transport models based only on the parton cascade, the elliptic flow has been shown to be sensitive to the parton scattering cross section, and a large value can be obtained with a large cross section [30].
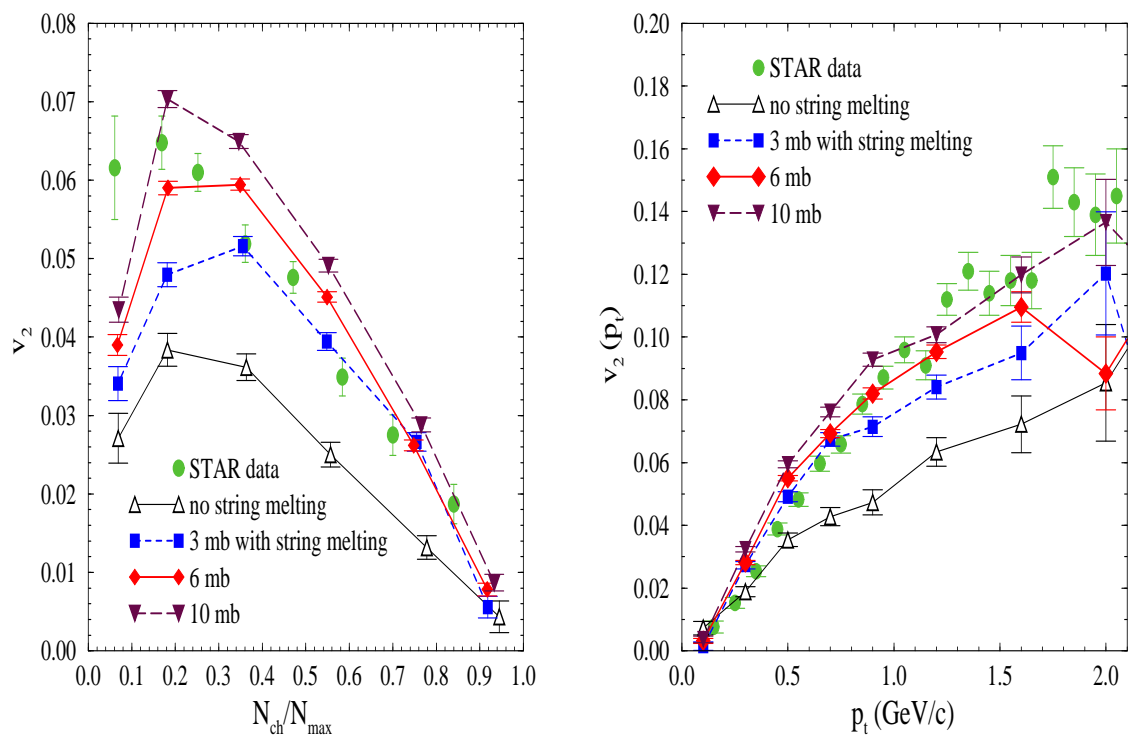

Fig. 3. Charged particle multiplicity (left panel) and transverse momentum (right panel) dependence of elliptic flow in $\mathrm{Au}+\mathrm{Au}$ collisions at $\sqrt{s}=130 \mathrm{~A} \mathrm{GeV}$. Data from the STAR collaboration [ 31] are shown by filled circles, while theoretical results for different partonic dynamics are given by curves.

Results on the charged particle elliptic flow from the AMPT model for $\mathrm{Au}+\mathrm{Au}$ collisions at center-of-mass energy of $130 A \mathrm{GeV}$ are shown in Fig. 3 as a function of both the charged particle multiplicity (left panel) and transverse momentum (right panel). Theoretical values from the default AMPT model (no string melting) are much smaller than that measured in the experiments by the STAR collaboration [ 31]. This is mainly due to the small fraction of energy that is carried by minijet partons and the lack of transverse collective motion of the strings. As a result, the elliptic flow is significantly reduced after minijet partons combine with strings and fragment to hadrons.

Since the initial energy density of produced matter in central $\mathrm{Au}+\mathrm{Au}$ collisions at RHIC is more than an order of magnitude higher than the critical energy density $\left(\sim 1 \mathrm{GeV} / \mathrm{fm}^{3}\right)$ expected for the formation of the quark-gluon plasma, keeping strings in the high energy density region thus underestimates the partonic effects in these collisions. To model the melting of strings into partons in regions of high energy density, we have extended the AMPT model to allow the initially excited strings to fragment into hadrons using the LUND fragmentation model and then 
convert these hadrons to their constituent quarks but with bare masses, i.e., a meson is converted to a quark and an anti-quark, while a baryon is converted to three quarks. We further assume that quarks are produced isotropically in the rest frame of a hadron and start to interact only after a proper formation time given by the inverse of the hadron transverse mass. Scatterings among these quarks are then treated using the parton cascade ZPC. After the quarks stop interacting, we model the hadronization by combining the nearest quark and antiquark into a meson and three quarks into a baryon with the same flavor. The resulting hadrons are given an additional formation time of $0.7 \mathrm{fm} / c$ in their rest frame and then imported to the ART hadronic transport model to take into account their rescatterings.

With strings converted to partons, the initial energy originally stored in strings also contributes to the parton dynamics. This leads to a larger elliptic flow and also a stronger dependence on the parton scattering cross section, making it possible to determine the strength of partonic interactions from the final elliptic flow. As shown in Fig. 3, a large parton scattering cross section of $6-10 \mathrm{mb}$ is needed to account for the observed dependence of charged particle elliptic flow on the total charged particle multiplicity and their transverse momentum.

\section{Pion Interferometry}

Particle interferometry based on the Hanbury-Brown Twiss (HBT) effect can provide information not only on the spatial extent of an emission source but also on its expansion velocity and emission duration [4, 32, 33]. In particular, the long emission time as a result of the quark-gluon plasma to hadronic matter phase transition in the initial stage of heavy ion collisions is expected to lead to a much larger radius parameter along the direction of the total momentum of detected two particles than that perpendicular to both this direction and the beam direction.

From the emission function, which is given by the space-time and momentum distribution of particles at freezeout in the AMPT model, we can evaluate the correlation function $C(\mathbf{Q}, \mathbf{K})$ of two identical pions including their Coulomb interaction. Here, $\mathbf{Q}$ and $\mathbf{K}$ are the relative and total momenta of the two pions. The six-dimension correlation function is usually shown as a function of the invariant momentum $\left(Q_{\text {inv }}=\sqrt{-Q^{2}}\right)$ or as a function of the projection of the relative momentum $\mathbf{Q}$ in the "out-side-long" (osl) system [4, 32], defined by the beam direction $\left(Q_{\text {long }}\right)$, the direction along the total transverse momentum of the two pions $\left(Q_{\text {out }}\right)$, and the direction orthogonal to the above two directions $\left(Q_{\text {side }}\right)$.

In the left panel of Fig. 1 , we show the one-dimensional projections of the correlation function for midrapidity pions with transverse momentum $125<p_{\perp}<$ $225 \mathrm{MeV} / \mathrm{c}$ in $\mathrm{Au}+\mathrm{Au}$ collisions at $\sqrt{s}=130 \mathrm{~A} \mathrm{GeV}$. Also shown is the measured $\pi^{-}$correlation function by the STAR collaboration without correcting the effect due to Coulomb interaction [34]. In evaluating the one-dimensional projection of the correlation function onto one of the $Q_{\text {out }}, Q_{\text {side }}, Q_{\text {long }}$ axes, we have integrated the other two $\mathbf{Q}$ components over the range $0-35 \mathrm{MeV} / c$. Dash-dotted curves 

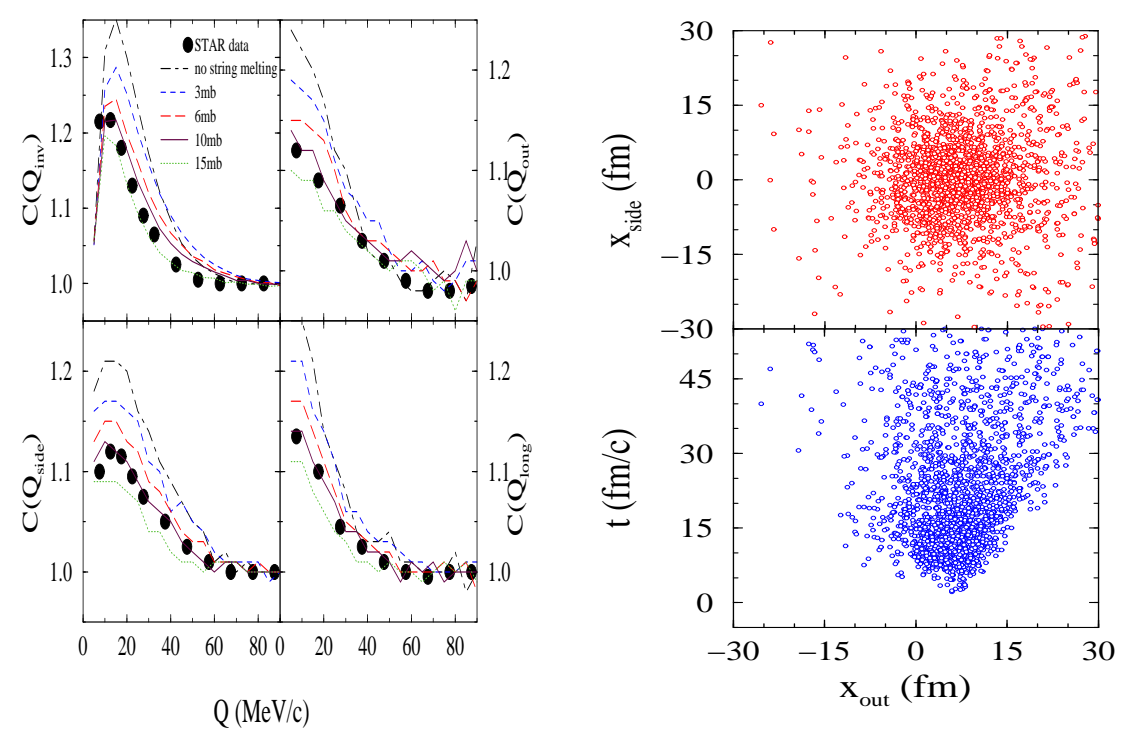

Fig. 4. Left panel:Correlation functions for midrapidity pions from central $\mathrm{Au}+\mathrm{Au}$ collisions at $\sqrt{s}=130 \mathrm{~A} \mathrm{GeV}$. Coulomb-uncorrected correlation functions from the STAR collaboration for low $p_{T} \pi^{-}$at midrapidity from central collisions are shown by filled circles. Dash-dotted curves are results from the default AMPT model, while other curves are from the extended AMPT model with string melting and various values for the parton scattering cross section. Right panel: Distribution of the emission source from the AMPT model for midrapidity pions with $125<p_{\perp}<225$ $\mathrm{MeV} / c$ in the $x_{\text {out }}-x_{\text {side }}$ (upper panel) and $x_{\text {out }}-t$ (lower panel) spaces.

in the figure are from the default AMPT model with a parton scattering cross section of $\sigma_{\mathrm{p}}=3 \mathrm{mb}$, while other curves are from the extended AMPT model with string melting but different values of parton scattering cross section. It is seen that with string melting both the width of the $Q_{\text {inv }}$ correlation function and its height decrease with increasing $\sigma_{\mathrm{p}}$. To reproduce the measured one-dimensional correlation functions from the STAR collaboration, we need a parton scattering cross section of about $10 \mathrm{mb}$ in the extended AMPT model with string melting.

In the right panel of Fig. 1, we show the distribution of the emission source from the AMPT model for midrapidity pions with $125<p_{\perp}<225 \mathrm{MeV} / c$ in the $x_{\text {out }}-x_{\text {side }}$ and $x_{\text {out }}-t$ spaces. It is seen that the emission source shows a large halo around a central core. The halo consists of not only pions from decays of long-lived resonances such as the $\omega$ but also thermal pions. The latter contribution to the halo becomes increasingly important when the collective expansion velocity of the source is large as a result of increasing parton cross section. The emission source also shows a strong correlation between $x_{\text {out }}$ and $t$, with the width of its $x_{\text {out }}$ 
distribution increasing with the emission time $t$.
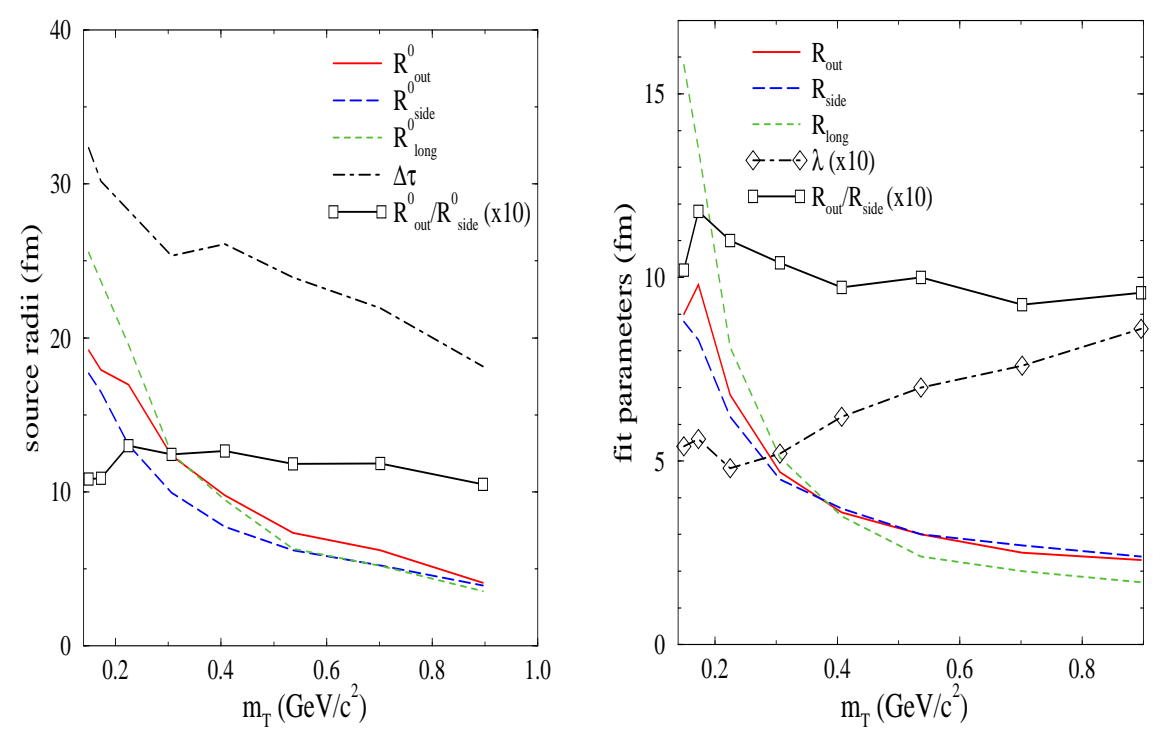

Fig. 5. Left panel: Radii $R_{\text {out }}^{0}, R_{\text {side }}^{0}$, and $R_{\text {long }}^{0}$ and emission duration $\Delta \tau$ of the emission source for midrapidity pions with $125<p_{\perp}<225 \mathrm{MeV} / c$ in central $\mathrm{Au}+\mathrm{Au}$ collisions at $\sqrt{s}=130 \mathrm{~A} \mathrm{GeV}$ as functions of the average transverse mass of the pion pair from the AMPT model with string melting and parton scattering cross section $\sigma_{\mathrm{p}}=15 \mathrm{mb}$. Right panel: Same as left panel for the radius parameters obtained from fitting the two-pion correlation by a Gaussion function in their relative momentum.

The size of emission source can be estimated from the curvature of the correlation function at $\mathbf{Q}=0$, i.e, the second moments of the emission function from the AMPT model. In the left panel of Fig. 5, we show the dependence of the source radii of midrapidity pions $(-0.5<y<0.5)$ on the average transverse mass $m_{\mathrm{T}}$ of the pion pair from the AMPT model with string melting and a parton cross section of $10 \mathrm{mb}$. At low transverse mass, all three radii $R_{\text {out }}^{0}, R_{\text {side }}^{0}$, and $R_{\text {long }}^{0}$ are large and have values between 10 and $25 \mathrm{fm} / \mathrm{c}$. The emission duration $\Delta \tau$ also lasts a long time of more than $25 \mathrm{fm} / \mathrm{c}$. As the average pion transverse mass increases, both the emission duration and the source radii, particularly $R_{\text {long }}^{0}$, are found to decrease. The ratio $R_{\text {out }}^{0} / R_{\text {side }}^{0}$ has a value of about 1.2 for most values of $m_{\mathrm{T}}$ and is smaller than the prediction based on the hydrodynamical model with freezeout treated via the hadronic transport model [35].

Empirically, radii of the emission source are obtained from fitting the measured correlation function by a Gaussian function in the relative momentum of the pion pair. Results from such a fit to the correlation function obtained from the AMPT 
model with string melting and $\sigma_{\mathrm{p}}=10 \mathrm{mb}$ are shown in the right panel of Fig. 5 as functions of the average pion transverse mass. The fitted radius parameters $R_{\text {out }}, R_{\text {side }}$, and $R_{\text {long }}$ are about a factor of two to three smaller than corresponding source radii evaluated directly from the emission function, indicating that the emission source from the AMPT model deviates appreciably from a Gaussian one. Furthermore, the ratio $R_{\text {out }} / R_{\text {side }}$ of the fitted Gaussian radius parameters is much closer to one than that from the source radii obtained from the emission function.

\section{Conclusions}

Using a multiphase transport model, which includes both initial partonic and final hadronic interactions, we have studied the rapidity distributions of charged particles such as protons, antiprotons, pions, and kaons in heavy ion collisions at RHIC. With the model parameters constrained by data from central $\mathrm{Pb}+\mathrm{Pb}$ collisions at $\sqrt{s}=17 A \mathrm{GeV}$ at SPS, theoretical results on the total charged particle multiplicity at midrapidity in central $\mathrm{Au}+\mathrm{Au}$ collisions at $\sqrt{s}=56 \mathrm{~A}$ and $130 \mathrm{~A} \mathrm{GeV}$ agree quite well with the data from the PHOBOS collaboration. Also, the predicted $\bar{p} / p$ ratio is consistent with that from the STAR and BRAHMS collaborations. Furthermore, the centrality dependence of the charged particle rapidity distribution measured by the BRAHMS collaboration is well reproduced. These hadronic observables are, however, less sensitive to the initial partonic interactions than to the final hadronic interactions. On the other hand, partonic effects are important for both the elliptic flow of charged particles and the two-pion correlation function. To reproduce the measured elliptic flow and two-pion correlation function by the STAR collaboration, we need not only to convert the initial strings to partons but also to use a large parton-parton scattering cross section. Our results thus demonstrate the possibility of studying the dynamics of partonic matter in heavy ion collisions at RHIC. To further test these partonic effects, it is necessary to study their dependence on the initial parton distribution, the inelastic scatterings in the partonic stage, and the mechanism for melting the initial strings as well as for hadronization. Such an improved study based on the AMPT model will be very useful in finding the signals for the quark-gluon plasma formed in heavy ion collisions at RHIC.

\section{Acknowledgments}

This talk is based on work supported by the National Science Foundation under Grant Nos. PHY-9870038 and PHY-0098805, the Welch Foundation under Grant No. A-1358, and the Texas Advanced Research Program under Grant No. FY99010366-0081.

\section{References}

1. E. Shuryak, Phys. Lett. B78 (1978) 150. 
C. M. Ko et al.

2. J. Rafelski and B. Müller, Phys. Rev. Lett. 48 (1982) 1066.

3. T. Matsui and H. Satz, Phys. Lett. B178 (1986) 416.

4. S. Pratt, Phys. Rev. D33 (1986) 1314.

5. O. Ollitrault, Phys. Rev. D46 (1992) 229.

6. X.N. Wang and M. Gyulassy, Phys. Rev. Lett. 68, (1992) 1480.

7. M. Asakawa, U. Heinz, and B. Müller, Phys. Rev. Lett. 85 (2001) 2072.

8. S. Jeon and V. Koch, Phys. Rev. Lett. 85 (2001) 2076.

9. Z.W. Lin and C.M. Ko, Phys. Rev. C64 (2001) 041901.

10. B. Zhang, C.M. Ko, B.A. Li, and Z.W. Lin, Phys. Rev. C61 (2000) 067901.

11. Z.W. Lin, S. Pal, C.M. Ko, B.A. Li, and B. Zhang, Phys. Rev. C64 (2001) 011902.

12. Z.W. Lin and C.M. Ko, Phys. Rev. C65 (2002) 034904.

13. S. Pal, C.M. Ko, and Z.W. Lin, nucl-th/016073.

14. B. Zhang, C.M. Ko, B.A. Li, Z.W. Lin, and B.H. Sa, Phys. Rev. C62 (2000) 054905; ibid. in press.

15. Z.W. Lin, C.M. Ko, and S. Pal, nucl-th/0204054.

16. M. Gyulassy and X.N. Wang, Comp. Phys. Comm. 83, (1994) 304.

17. A.H. Mueller and J. Qiu, Nucl. Phys. B268, (1986) 427.

18. M. Gyulassy and X.N. Wang, Nucl. Phys. B420 (1994) 583.

19. B. Zhang, Comp. Phys. Comm. 109 (1998) 193.

20. T. Sjöstrand, Comp. Phys. Comm. 82 (1994) 74.

21. B.A. Li and C.M. Ko, Phys. Rev. C52 (1995) 2037.

22. C.M. Ko and R. Yuan, Phys. Lett. B192 (1987) 31.

23. H. Appelshäuser et al. (NA49 Collaboration), Phys. Rev. Lett. 82 (1999) 2471.

24. B.B. Back et al. (PHOBOS Collaboration), Phys. Rev. Lett. 85 (2000) 3100.

25. C. Adler et. al. (STAR collaboration), Phys. Rev. Lett. 86 (2001) 4778; I.G. Bearden et al. (BRAHMS collaboration), ibid. 87 (2001) 112301.

26. K. Adcox et al., (PHENIX Collaboration), Phys. Rev. Lett., in press.

27. I.G. Bearden et al. (BRAHMS Collaboration), Phys. Lett. B523 (2001) 227.

28. X.N. Wang and M. Gyulassy, Phys. Rev. Lett. 86 (2001) 3496.

29. H. Sorge, Phys. Rev. Lett. 78 (1997) 2309.

30. B. Zhang, M. Gyulassy and C.M. Ko, Phys. Lett. B455 (1999) 45.

31. K.H. Ackermann et al. (STAR Collaboration), Phys. Rev. Lett. 86 (2001) 402.

32. G.Bertsch, M. Gong and M. Tohyama, Phys. Rev. C37 (1988) 1896.

33. D.H. Rischke and M. Gyulassy, Nucl. Phys. A608 (1996) 479.

34. C. Adler et al., (STAR Collaboration), Phys. Rev. Lett. 87 (2001) 082301.

35. S. Soff, S.A. Bass and A. Dumitru, Phys. Rev. Lett. 86 (2001) 3981. 